\title{
Antiviral Therapy
}

National Cancer Institute

\section{Source}

National Cancer Institute. Antiviral Therapy. NCI Thesaurus. Code C16119.

Treatment of viral infections with agents that prevent viral replication in infected cells without impairing the host cell function. 